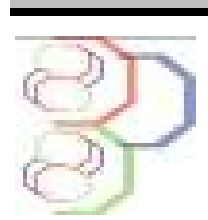

Journal of Applied Biosciences 106:10215 -10223

ISSN 1997-5902

\title{
Le bois du frake issu de plantation exprime aussi de bonnes qualités technologiques
}

\author{
Assandé AHOBA ${ }^{1 *}$, Auguste Emmanuel ISSALI ${ }^{2}$, Lewis Carol GNAORÉ ${ }^{3}$, Arsène Gondo GBOGBO³, \\ Martin Kouassi KOUADIO 4 \\ 1 Station SRT Centre National de Recherche Agronomique (CNRA), 08 BP 33 Abidjan 08. \\ 2 Station de Recherche sur le Cocotier Port Bouët Marc Delorme, CNRA, 07 BP 13 Abidjan 07, Côte d'Ivoire. \\ ${ }^{3}$ ONG, Solidaridad, 25 BP 1108 Abidjan 25. \\ ${ }^{4}$ Société de Développement des Forêts (SODEFOR), 01 BP 3770 Abidjan 01. \\ ${ }^{*}$ Corresponding Author: ahauba@yahhoo.fr
}

Original submitted in on 31st August 2016. Published online at www.m.elewa.org on 31 $31^{\text {st }}$ October 2016

http://dx.doi.org/10.4314/jab.v106i1.1

\section{RESUME}

Objectif: Les qualités technologiques du bois mature de Fraké issu de plantation sont mal connues à la différence de celles du bois issu de forêt naturelle. Le travail réalisé vise à montrer la ressemblance entre les Frakés de plantation et ceux de forêt naturelle en ce qui concerne les qualités technologiques de leurs bois. Méthodologie et résultats : Six arbres, âgés de 32 ans, issus d'un peuplement de Fraké artificiellement planté, ont été sélectionnés, abattus et soumis aux tests technologiques de leurs bois. Les résultats montrent qu'à 32 ans, le Fraké de plantation produit des billes de bonne rectitude dont les dimensions sont relativement élevées en longueurs (13 à $21 \mathrm{~m}$ ) et en diamètres $(25$ à $50 \mathrm{~cm}$ ) par rapport aux Frakés de forêt naturelle. Certains arbres comme le Fraké3 dans le cas de notre étude, affichent des diamètres particulièrement intéressants pour une exploitation en bois d'œuvre. Le sciage est facile et donne un bon rendement avec des débités de largeurs intéressantes pour les échanges commerciaux. Les qualités physiques et mécaniques montrent une tendance à la baisse avec la hauteur de l'arbre à partir de $6 \mathrm{~m}$.

Conclusion et applications : Les Frakés de plantation expriment des aptitudes technologiques similaires à celles de forêt naturelle. En conséquence, ils peuvent être utilisés sous forme de bois d'œuvre au même titre que les Frakés de forêt naturelle.

Mots-clés : Fraké de plantation, cubage, sciage, qualité physique et mécanique, Côte d'Ivoire

\section{ABSTRACT}

Objective: Technological qualities of the mature wood of Terminalia superba (Fraké) from plantation are badly known unlike those from wood from natural forest. Achieved work aimed to show the likeness between Frakés from plantation and those from natural forest regarding technological properties of their wood.

Methodology and results: Six trees, aged thirty-two, from artificially planted Fraké standing forest, were selected, felled and subjected to technological test of their woods. Results showed that at 32 years old, Frakés from plantation yield logs expressing good straightness whose dimensions are relatively high in length (from 13 to $21 \mathrm{~m}$ ) and in diameters (from 25 to $50 \mathrm{~cm}$ ) compared to Frakés from natural forest. Some trees like Frake 3 in the case of our study showed interesting diameters for logs exploitation. The sawing is easy and gives good 
yield with lumbers showing interesting width for commercial transactions. Physical and mechanic properties tend to decrease when height becomes greater than or equal to $6 \mathrm{~m}$.

Conclusion et applications: Plantation Frakés express the same technological properties than those from natural forest. Therefore, they can be used in the form of lumber in the same manner as the natural ones.

\section{INTRODUCTION}

Le Fraké est l'une des plus importantes essences forestières utilisées en reboisement par la Côte d'Ivoire. Après avoir occupé la première place pendant de nombreuses années en termes de superficie plantée, cette essence a été supplantée par le Teck et reléguée à la deuxième place des essences forestières régénérées artificiellement. La superficie couverte en 2009 est de 25824 ha soit environ $14 \%$ des plantations forestières de la Côte d'Ivoire (SODEFOR, 2012). Jusqu'à une époque relativement récente, seule les grumes issues de forêts naturelles étaient valorisées. II s'en est suivi un appauvrissement en quantité et en qualité de la ressource qui a amené le gouvernement à mettre en place de nombreuses plantations artificielles. Les premiers essais de qualification technologique du

\section{MATERIEL ET METHODES}

Site d'étude: L'étude a été réalisée à Mopri, géographiquement localisé dans la région administrative des lagunes, entre $5^{\circ} 40^{\prime}$ et $5^{\circ} 55^{\prime}$ de latitude Nord et entre $4^{\circ} 52^{\prime}$ et $5^{\circ} 02^{\prime}$ de longitude Ouest. L'échantillonnage retenu est composé d'un lot de 6 arbres de Frakés asymptomatiques (Figure 1), récoltés en 2014 dans la parcelle référencée 117-81/01 de la Société de Développement des Forêts (SODEFOR).

Cubage et cotation des billons : Les billes issues des arbres abattus à la tronçonneuse ont été transportées à l'usine avant d'être découpées en billons de longueurs réduites à $2,5 \mathrm{~m}, 3 \mathrm{~m}$ et $3,5 \mathrm{~m}$ de la base au sommet de chaque arbre. Ces billons ont été individuellement cubés et cotés. La cotation résumée dans le tableau 1, est une appréciation qualitative de chaque billon à partir d'un ensemble de critères affectés d'une échelle de notation allant de 1 à 3 . Ces critères portent sur la rectitude, les contreforts, les méplats, la section du billon, les cannelures, les fentes, la position du cœur, l'état sanitaire du cœur, les altérations superficielles, les nœuds ou bosses.

Sciage et rendement sciage des billons : Le sciage à plat conformément aux spécifications de l'usine participant à l'étude, a été réalisé sur l'une des deux lignes LT300 destinées aux grumes de gros diamètres bois issu de ces plantations ont porté sur des sujets relativement jeunes âgés d'une quinzaine d'années (Durand, 1985; Durand et Edi, 1979). Les conclusions de ces essais montrent de meilleures qualités de bois en faveur des Frakés issus de forêt naturelle. Aujourd'hui, la disponibilité de peuplements âgés, en cours d'exploitation, donne la possibilité de procéder à de nouveaux essais de détermination des valeurs au plan technologique pour cette essence. Ainsi, les bois de Fraké naturel et artificiel sont-ils comparables? Telle est la préoccupation à laquelle la présente étude vise à répondre et valoriser technologiquement le bois artificiel de Fraké en recherchant la ressemblance entre ce dernier et celui issu de forêt naturelle.

de l'usine. Cette ligne à pilotage automatique comporte une scie de tête horizontale (Figure 2) à bande étroite (32-50 $\mathrm{mm}$ de largeur et $2 \mathrm{~mm}$ d'épaisseur) de marque Wood Mizer équipée de rayons laser pour le passage du trait de scie. Un système d'arrosage haute pression maintient la lame lubrifiée à l'eau et accroît les performances générales de cette lame. Dans la technologie adoptée par l'unité, la grume est fixe et c'est la scie de tête qui se déplace en va et vient enlevant à chaque passage une planche d'épaisseur désirée. Un plateau central diamétral d'épaisseur $5 \mathrm{~cm}$ a été prélevé par billon et retenu pour l'étude des propriétés physiques et mécaniques conformément aux normes d'essais (Sallenave, 1955, 1964, 1971 ; AFNOR, 1988). Dans le cadre de cette étude, deux mesures ont été systématiquement réalisées par arbre pour les propriétés physiques et mécaniques. Des mesures de quelquesunes des caractéristiques physiques et mécaniques ont également été réalisées en fonction de la hauteur de prélèvement de l'échantillon dans l'arbre. Les sciages obtenus ont été délignés et cubés pour accéder au rendement sciage. Quelques observations d'aspects en termes de coloration, figurations ou veines du bois ont été faites sur les planches. 
Ahoba et al., J. Appl. Biosci. 2016 Le bois du frake issu de plantation exprime aussi de bonnes qualités
technologiques

Tableau 1 : Critères de cotation des billons destinés au sciage

\begin{tabular}{|l|l|l|l|}
\hline \multicolumn{2}{|c|}{ Cote } & \multicolumn{1}{c|}{$\mathbf{2}$} & \\
\hline Rectitude du fût & Droit & & \multicolumn{1}{c|}{$\mathbf{3}$} \\
\hline Contrefort & pas de contrefort & légère courbure & courbure prononcée \\
\hline Cannelure & pas de cannelure & légère contrefort & contrefort important \\
\hline Méplats & pas de méplat & méplat peu marquee & grosse cannelure \\
\hline Section & Circulaire & ovale & méplat marquee \\
\hline Fentes & pas de fente & légères fentes & triangulaire, carré ou autres \\
\hline Position du cœur & cœur centré & cœur légèrement excentré & nombreuses fentes prononcées \\
\hline Etat sanitaire du cœur & cœur sain & $\begin{array}{l}\text { légère pourriture ou attaque de } \\
\text { termite }\end{array}$ & $\begin{array}{l}\text { pourriture marquée ou attaque } \\
\text { profonde par les termites }\end{array}$ \\
\hline Altérations & $\begin{array}{l}\text { pas d'attaque sur le } \\
\text { roulant }\end{array}$ & $\begin{array}{l}\text { légère attaque (pourriture, } \\
\text { piqûre, termite) }\end{array}$ & $\begin{array}{l}\text { attaque prononcée (pourriture, } \\
\text { piqûre, termite) }\end{array}$ \\
\hline Nœuds ou bosses & pas de nœud apparent & au plus 2 nœuds apparents & plus de 2 nœuds apparents \\
\hline
\end{tabular}

Analyse des données : Les données collectées sur les 6 arbres ont été traitées avec les logiciels XIstat et SPSS, respectivement les versions 2007 et 16.0. L'analyse de variance selon la procédure multivariée GLM (Generalized Linear Models), supportant les écarts à la

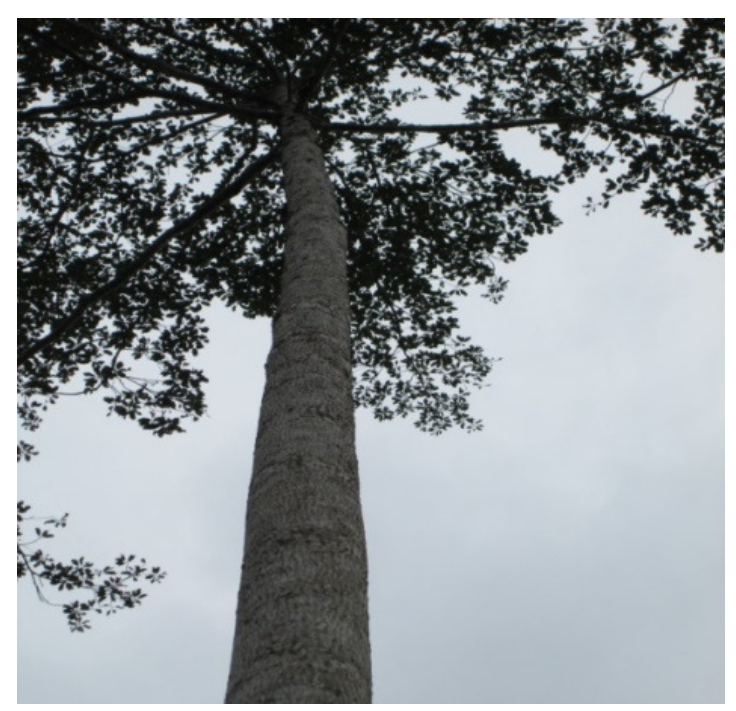

Figure 1 : Fraké visuellement asymptomatique dans une plantation artificielle en forêt dense semi-décidue de Mopri

\section{RÉSULTATS}

Cubage des billons: Pour tous les 3 diamètres mesurés, 2 classes distinctes de moyennes ont été identifiées. La première $\mathrm{C} 1$, représentée par le Fraké 5 , est caractérisée par de faibles diamètres. La seconde $\mathrm{C} 2$, illustrée par le Fraké 3 , est marquée par des diamètres élevés (Tableau $2.1 ; 2.2$. et 2.3.). Le cubage des arbres normalité, a été utilisée. Néanmoins, l'égalité des variances, des sous populations analysées, a été vérifiée. Cette analyse a été assortie de la comparaison de moyennes selon le test de Newman-Keuls au seuil de confiance de $95 \%$.

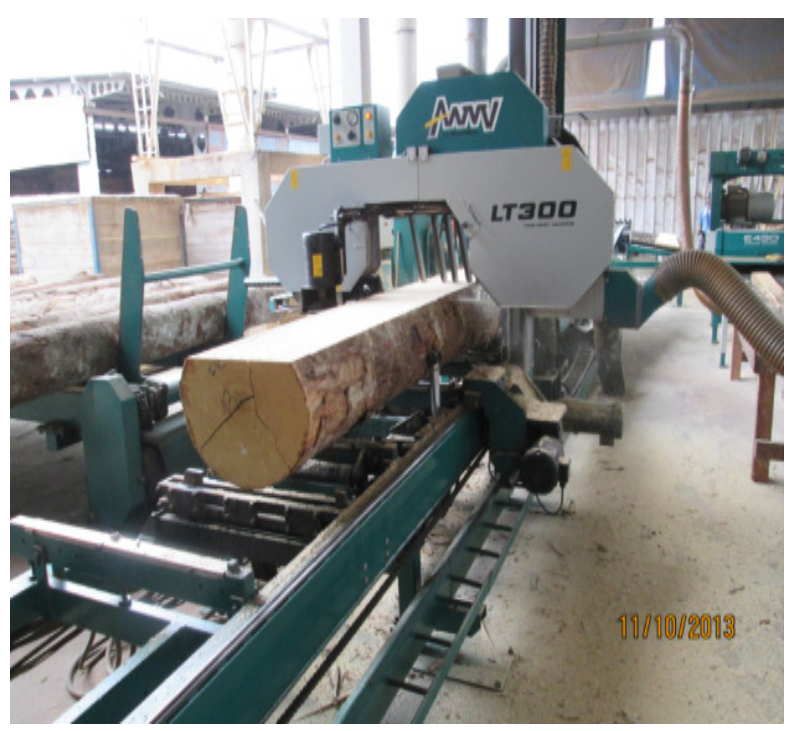

Figure 2 : Scie horizontale de marque "Wood Mizer" utilisée dans l'expérimentation

consigné dans les tableaux $2.3 ; 2.4$ permet de noter les valeurs obtenues en diamètre moyen, en longueur de grume, volume grume et en nombre de billons. Le découpage des billes de la base au sommet a permis l'obtention de 33 billons. Le plus grand nombre de billons obtenu est 7 avec les arbres étiquetés Fraké 3 et Fraké 4. 
II ressort de ces valeurs que les Frakés de 32 ans, issus de plantation, présentent des dimensions de billes relativement élevées en longueurs de fût utile (13 à 21 $\mathrm{m}$ ) et en diamètres ( 30 à $50 \mathrm{~cm}$ au gros bout et 25 à 48 $\mathrm{cm}$ au petit bout). Le volume total prélevé de $10,460 \mathrm{~m}^{3}$ pour les 6 arbres conduit par calcul à un volume moyen de $1,800 \mathrm{~m}^{3}$ par arbre.

Tableau 2: Classification des moyennes des 3 diamètres mesurés et cubage des arbres échantillonnés

\begin{tabular}{|c|c|c|c|c|c|c|c|}
\hline $\begin{array}{l}\text { 1. Variable } \\
\text { dépendante }\end{array}$ & Individu & Moyenne & CV (\%) & $\begin{array}{l}\text { 2. Variable } \\
\text { dépendante }\end{array}$ & Individu & Moyenne & CV (\%) \\
\hline \multirow{6}{*}{ Diagrobo } & Fraké 5 & $33,000 a$ & 5,87 & Diapebo & Fraké 5 & $31,400 a$ & 6,61 \\
\hline & Fraké 2 & $36,600 \mathrm{ab}$ & 5,29 & & Fraké 2 & $33,800 a b$ & 6,14 \\
\hline & Fraké 1 & $37,000 \mathrm{ab}$ & 5,85 & & Fraké 4 & $34,571 a b$ & 5,07 \\
\hline & Fraké 4 & $37,143 a b$ & 4,41 & & Fraké 1 & $35,000 a b$ & 6,63 \\
\hline & Fraké 6 & $40,000 \mathrm{ab}$ & 5,42 & & Fraké 6 & $37,000 a b$ & 6,27 \\
\hline & Fraké 3 & $43,429 b$ & 3,77 & & Fraké 3 & $40,714 b$ & 4,31 \\
\hline $\begin{array}{l}\text { 3. Variable } \\
\text { dépendante }\end{array}$ & Individu & Moyenne & CV (\%) & 4. Individu & $\begin{array}{l}\text { Longueur } \\
\text { grume }(\mathrm{m})\end{array}$ & $\begin{array}{c}\text { Volume } \\
\text { grume }\left(\mathrm{m}^{3}\right)\end{array}$ & $\begin{array}{l}\text { Nombre de billons } \\
(2,5 \text { à } 3,5 \mathrm{~m})\end{array}$ \\
\hline \multirow[t]{6}{*}{ Diamoy } & Fraké 5 & $31,800 a$ & 6,28 & Fraké 1 & 13 & 1,252 & 4 \\
\hline & Fraké 2 & $35,000 a b$ & 5,70 & Fraké 2 & 15 & 1,459 & 5 \\
\hline & Fraké 4 & $35,429 a b$ & 4,76 & Fraké 3 & 21 & 2,941 & 7 \\
\hline & Fraké 1 & $35,500 \mathrm{ab}$ & 6,28 & Fraké 4 & 21 & 2,008 & 7 \\
\hline & Fraké 6 & $38,250 \mathrm{ab}$ & 5,83 & Fraké 5 & 15 & 1,212 & 5 \\
\hline & Fraké 3 & $41,714 b$ & 4,04 & Fraké 6 & 15 & 1,588 & 5 \\
\hline
\end{tabular}

Légende : Diagrobo : Diamètre gros bout $(\mathrm{cm})$. Diapebo : Diamètre petit bout $(\mathrm{cm})$. Diamoy : Diamètre moyen $(\mathrm{cm})$.

Cotation des billons: Les cotations qualitatives (Tableau 3) indiquent que les Frakés de plantation présentent à $94 \%$ des billons de bonne rectitude avec au plus deux nœuds apparents dans $21 \%$ des cas. Les contreforts, méplats et altérations sont quasi inexistants.
La section du billon est plutôt circulaire (Figure 3) avec de légères fentes et un cœur centré dans un état sanitaire satisfaisant dans $97 \%$ des cas. L'aubier n'est pas discernable et forme un ensemble homogène avec le bois parfait

Tableau 3 : Cotation qualitative des billons

\begin{tabular}{|l|l|l|l|l|l|l|l|l|l|}
\hline Critères & $\begin{array}{l}\text { Recti- } \\
\text { tude } \\
(\%)\end{array}$ & $\begin{array}{l}\text { Contre- } \\
\text { forts }(\%)\end{array}$ & $\begin{array}{l}\text { Méplat } \\
(\%)\end{array}$ & $\begin{array}{l}\text { Section } \\
(\%)\end{array}$ & $\begin{array}{l}\text { Fente } \\
(\%)\end{array}$ & $\begin{array}{l}\text { Position } \\
\text { cœur } \\
(\%)\end{array}$ & $\begin{array}{l}\text { Etat } \\
\text { sanitaire } \\
(\%)\end{array}$ & $\begin{array}{l}\text { Altéra- } \\
\text { tions } \\
(\%)\end{array}$ & $\begin{array}{l}\text { Nœuds } \\
(\%)\end{array}$ \\
\hline 1 & 93,93 & 93,93 & 81,82 & 84,85 & 90,91 & 96,97 & 96,97 & 96,97 & 78,79 \\
\hline 2 & 6,07 & 6,07 & 18,18 & 15,15 & 9,09 & 3,03 & 3,03 & 3,03 & 21,21 \\
\hline 3 & & & & & & & & & \\
\hline
\end{tabular}

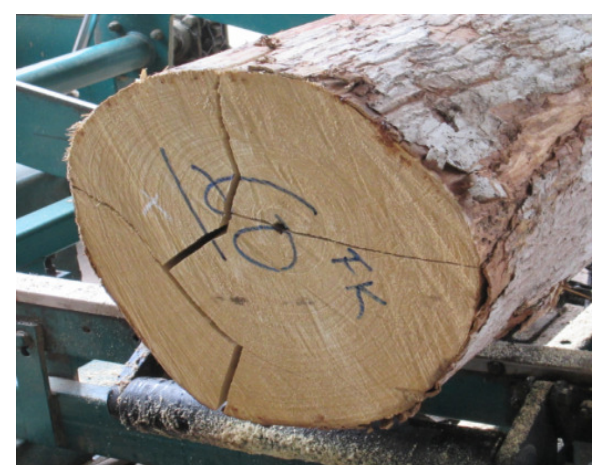

Figure 3 : Section d'un billon de Fraké avec des fentes et un cœur centré 
Sciage des billons: Le sciage à plat réalisé conformément aux spécifications de l'usine, a permis d'obtenir 374 débités de différentes épaisseurs (30, 35, 40,45 et $50 \mathrm{~mm}$ ) dont 35 ont été déclassés. L'ensemble des débités obtenus pour les six arbres, conduit à un nombre moyen de 62 débités par arbre. La répartition de ces débités par largeur indépendamment des épaisseurs
(Figure 4) fait ressortir 148 débités de largeur $14 \mathrm{~cm}, 80$ de largeur $19 \mathrm{~cm}, 69$ de largeur $24 \mathrm{~cm}$ et 42 de largeur 30 $\mathrm{cm}$. II ressort de ces résultats que les largeurs $140 \mathrm{~mm}$ sont les plus fréquentes et correspondent à $39 \%$ de l'ensemble des débités. Les largeurs supérieures ou égales à $150 \mathrm{~mm}$ sont majoritaires avec un pourcentage de $56 \%$ des débités.

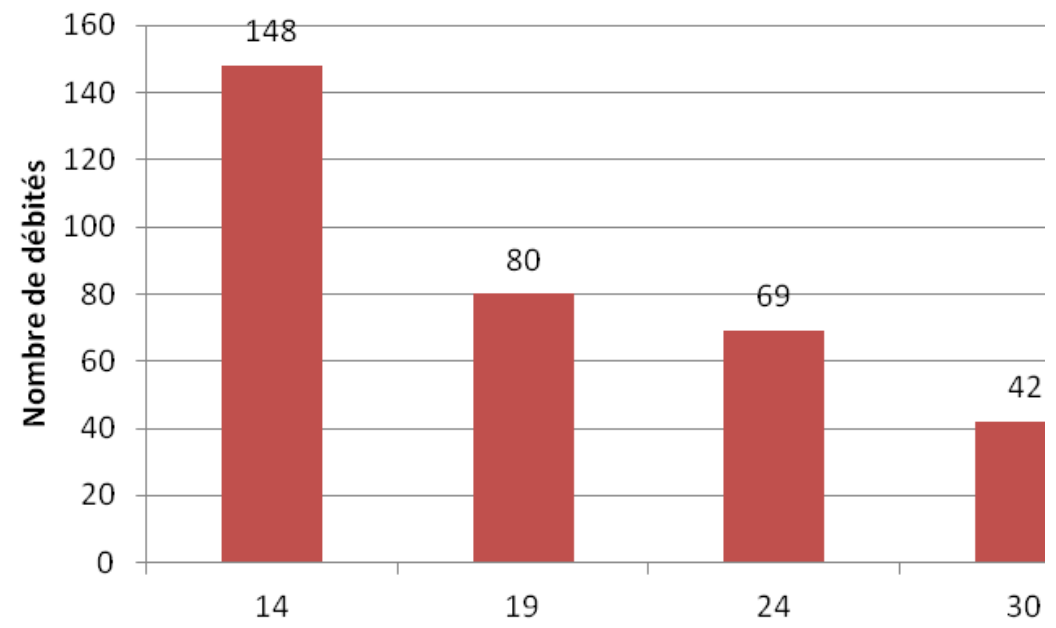

Largeurs de débités en $\mathrm{cm}$

Figure 4 : Nombre de débités par largeur indépendamment des épaisseurs

Rendement sciage : Le rendement obtenu en sciage sur le lot de 33 billons étudiés est de $64 \%$ avec le volume des déclassés et de $59 \%$ sans le volume des déclassés (Tableau 4).

Tableau 4 : Rendements sciage des billons

\begin{tabular}{|l|l|l|l|l|l|}
\hline \multirow{2}{*}{ Individus } & \multicolumn{5}{|c|}{ Paramètres } \\
\cline { 2 - 6 } & $\begin{array}{l}\text { Volume des } \\
\text { billons }\left(\mathrm{m}^{3}\right)\end{array}$ & $\begin{array}{l}\text { Volume des } \\
\text { débités }\left(\mathrm{m}^{3}\right)\end{array}$ & $\begin{array}{l}\text { Rendement } \\
\text { brut sciage }(\%)\end{array}$ & $\begin{array}{l}\text { Volume des planches } \\
\text { déclassées }\left(\mathrm{m}^{3}\right)\end{array}$ & $\begin{array}{l}\text { Rendement net } \\
(\%)\end{array}$ \\
\hline Ensemble des 6 arbres & 10,46 & 6,682 & 64 & 0,468 & 59 \\
\hline Par arbre & 1,800 & 1,114 & - & - & - \\
\hline
\end{tabular}

Aspect du bois : Le bois est d'aspect blanc crème avec parfois des figurations (Figure 5), qui donnent une certaine décoration au bois. Pour certains sujets, le bois présente un assemblage de diverses couleurs grisâtres (Figure 6), veinées de brun noirâtre parcourant la longueur de la planche. Cet assemblage de couleurs, souvent désigné par l'appellation "Fraké bariolé", confère au bois une esthétique particulièrement appréciée par les consommateurs qui doivent y mettre le prix pour l'acquérir. 


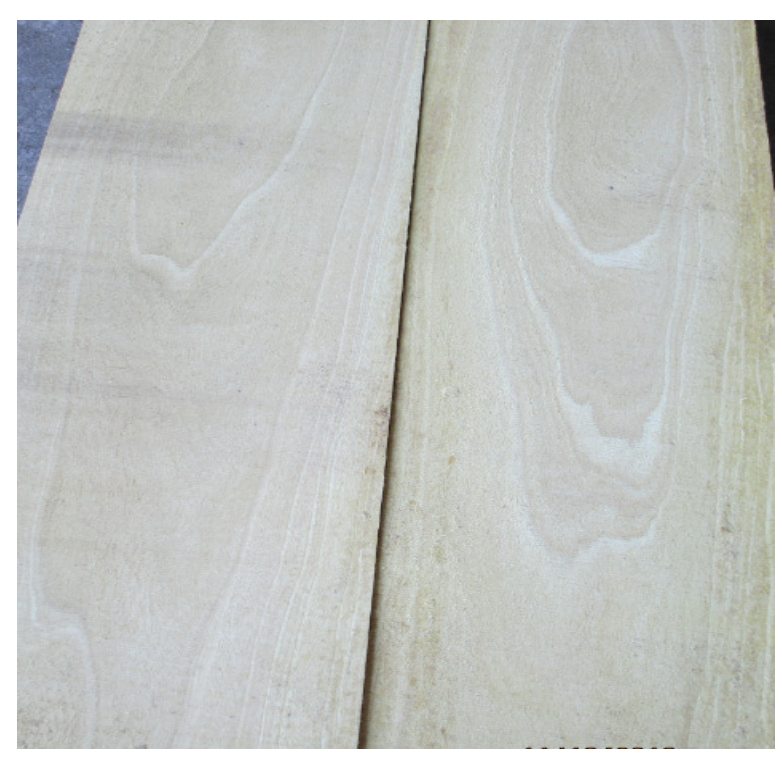

Figure 5 : Coloration blanc laiteux du bois de Fraké avec des figurations

Qualités physiques et mécaniques: Les caractéristiques physiques et mécaniques obtenues sont respectivement résumées dans les tableaux 5 et 6 . La densité moyenne de $456 \mathrm{~g} / \mathrm{dm}^{3}$ correspondant à 0,456 $\mathrm{kg} / \mathrm{m}^{3}$ (Tableau 5) présente un faible coefficient de variation qui dénote d'une certaine homogénéité d'un arbre à l'autre. II en est de même pour les autres caractéristiques physiques comme le retrait volumique, le coefficient de retrait volumique, le point de saturation et l'hygroscopicité. Pour les caractéristiques mécaniques (Tableau 6), les moyennes obtenues en flexion statique

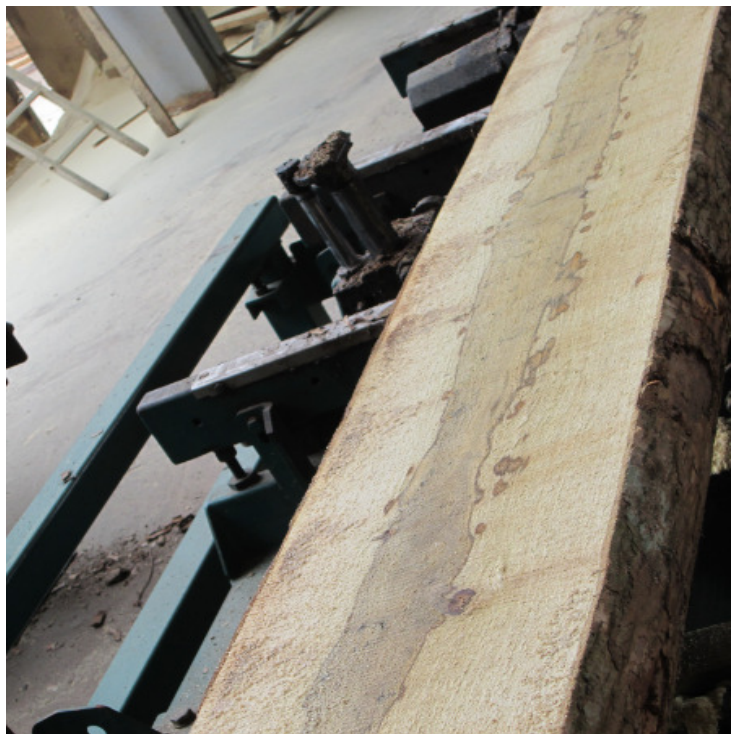

Figure 6 : Planche de Fraké bariolé

(68 MPa), en compression axiale (32 MPa) et en flexion dynamique $\left(0,19 \mathrm{Kgm} / \mathrm{cm}^{2}\right)$ sont peu variables d'un arbre à l'autre. Le tableau 7 présente la variation de quelques caractéristiques physiques et mécaniques en fonction de la hauteur de prélèvement de l'échantillon dans l'arbre. II découle de ce tableau 7 que les Frakés de 32 ans apparemment sains, montrent globalement une tendance à la baisse de de leurs caractéristiques à partir de $6 \mathrm{~m}$ de hauteur. Les contraintes admissibles en flexion statique semblent faire exception.

Tableau 5 : Caractéristiques physiques du Fraké de plantation en comparaison de celles du Fraké de forêt naturelle

\begin{tabular}{|c|c|c|c|c|c|}
\hline \multirow[b]{2}{*}{ Individus } & \multicolumn{5}{|c|}{ Paramètres } \\
\hline & $\begin{array}{c}\text { Densité D12 } \\
\left(\mathrm{g} / \mathrm{dm}^{3}\right)\end{array}$ & $\begin{array}{c}\text { Retrait } \\
\text { volumique } \\
\text { Total B\% }\end{array}$ & $\begin{array}{l}\text { Coefficient de } \\
\text { retrait volumique } \\
\text { v\% }\end{array}$ & $\begin{array}{c}\text { Point de } \\
\text { saturation S\% }\end{array}$ & Hygroscopicité d \\
\hline Fraké 1 & $\begin{array}{l}465,45 \\
383,83\end{array}$ & $\begin{array}{l}12,76 \\
11,88\end{array}$ & $\begin{array}{l}0,49 \\
0,44\end{array}$ & $\begin{array}{l}26,17 \\
26,91\end{array}$ & $\begin{array}{l}0,0024 \\
0,0021\end{array}$ \\
\hline Fraké 2 & $\begin{array}{l}507,55 \\
452,34\end{array}$ & $\begin{array}{l}12,11 \\
12,67\end{array}$ & $\begin{array}{l}0,38 \\
0,44\end{array}$ & $\begin{array}{l}32,10 \\
29,08\end{array}$ & $\begin{array}{l}0,0031 \\
0,0025\end{array}$ \\
\hline Fraké 3 & $\begin{array}{l}453,12 \\
497,61\end{array}$ & $\begin{array}{l}11,60 \\
10,87\end{array}$ & $\begin{array}{l}0,39 \\
0,33\end{array}$ & $\begin{array}{l}29,62 \\
32,78\end{array}$ & $\begin{array}{l}0,0027 \\
0,0033\end{array}$ \\
\hline Fraké 4 & $\begin{array}{l}468,66 \\
368,41\end{array}$ & $\begin{array}{c}11,14 \\
9,40\end{array}$ & $\begin{array}{l}0,45 \\
0,28\end{array}$ & $\begin{array}{l}24,94 \\
33,63\end{array}$ & $\begin{array}{l}0,0026 \\
0,0026\end{array}$ \\
\hline Fraké 5 & $\begin{array}{l}438,34 \\
374,49\end{array}$ & $\begin{array}{l}13,11 \\
12,87\end{array}$ & $\begin{array}{l}0,45 \\
0,36\end{array}$ & $\begin{array}{l}28,84 \\
35,51\end{array}$ & $\begin{array}{l}0,0024 \\
0,0023\end{array}$ \\
\hline Fraké 6 & $\begin{array}{l}504,48 \\
466,97\end{array}$ & $\begin{array}{l}11,12 \\
10,13\end{array}$ & $\begin{array}{l}0,34 \\
0,31\end{array}$ & $\begin{array}{l}32,26 \\
32,60\end{array}$ & $\begin{array}{l}0,0033 \\
0,0032\end{array}$ \\
\hline
\end{tabular}




\begin{tabular}{|c|c|c|c|c|c|}
\hline $\begin{array}{l}\text { Ahoba et al., } \\
\text { technologiques }\end{array}$ & J. Appl. Biosc & 016 Le bois & frake issu de $p$ & tion exprime a & e bonnes qualit \\
\hline Moyenne & $\begin{array}{c}\text { 456,30 Très } \\
\text { léger }\end{array}$ & 11,64 Moyen & $\begin{array}{c}0,39 \\
\text { Moyennement } \\
\text { nerveux }\end{array}$ & 30,37 Normal & 0,0027 Normale \\
\hline Cv \% & 10 & 0,55 & 16,75 & 10,78 & 14,81 \\
\hline $\begin{array}{l}\text { Intervalle de } \\
\text { confiance } 95 \%\end{array}$ & $456,30 \pm 31$ & $11,64 \pm 0,73$ & $0,39 \pm 0,04$ & $30,37 \pm 2,08$ & $0,0027 \pm 0,0003$ \\
\hline $\begin{array}{l}\text { Fraké naturel } \\
\text { Congo* }\end{array}$ & 450 Très léger & 10,7 Moyen & $\begin{array}{c}0,44 \\
\text { Moyennement } \\
\text { nerveux }\end{array}$ & 24 Normal & 0,0026 Normale \\
\hline $\begin{array}{l}\text { Fraké naturel } \\
\text { Gabon* }\end{array}$ & 480 Très léger & 11,7 Moyen & $\begin{array}{c}0,38 \\
\text { Moyennement } \\
\text { nerveux }\end{array}$ & 31 Normal & 0,0034 Normale \\
\hline $\begin{array}{l}\text { Fraké naturel } \\
\text { Cameroun* }\end{array}$ & 520 Léger & 9,1 Faible & $\begin{array}{c}0,39 \\
\text { Moyennement } \\
\text { nerveux }\end{array}$ & 24 Normal & 0,0033 Normale \\
\hline $\begin{array}{l}\text { Fraké naturel } \\
\text { Côte d'lvoire* }\end{array}$ & 550 Léger & 11 Moyen & $\begin{array}{c}0,43 \\
\text { Moyennement } \\
\text { nerveux }\end{array}$ & 26 Normal & 0,0030 Normale \\
\hline
\end{tabular}

${ }^{*}$ Source : CTFT, 1986

Tableau 6 : Caractéristiques mécaniques du bois de Fraké de plantation en comparaison de celles du Fraké de forêt naturelle

\begin{tabular}{|c|c|c|c|}
\hline \multirow[b]{2}{*}{ Individus } & \multicolumn{3}{|c|}{ Paramètres } \\
\hline & Flexion statique (MPa) & $\begin{array}{c}\text { Compression axiale } \\
\text { (MPa) }\end{array}$ & Flexion dynamique $\mathrm{Kgm} / \mathrm{cm}^{2} \mathrm{~K}$ \\
\hline Fraké 1 & $\begin{array}{c}94,35 \\
50\end{array}$ & $\begin{array}{l}43,92 \\
24,27\end{array}$ & $\begin{array}{l}0,21 \\
0,11\end{array}$ \\
\hline Fraké 2 & $\begin{array}{l}74,06 \\
49,85\end{array}$ & $\begin{array}{l}31,78 \\
26,91\end{array}$ & $\begin{array}{l}0,16 \\
0,16\end{array}$ \\
\hline Fraké 3 & $\begin{array}{l}80,55 \\
50,05\end{array}$ & $\begin{array}{l}35,01 \\
25,04\end{array}$ & $\begin{array}{l}0,36 \\
0,09\end{array}$ \\
\hline Fraké 4 & $\begin{array}{l}75,22 \\
49,80\end{array}$ & $\begin{array}{c}36 \\
24,85\end{array}$ & $\begin{array}{l}0,27 \\
0,13\end{array}$ \\
\hline Fraké 5 & $\begin{array}{l}79,68 \\
67,36\end{array}$ & $\begin{array}{l}32,95 \\
30,50\end{array}$ & $\begin{array}{l}0,22 \\
0,13\end{array}$ \\
\hline Fraké 6 & $\begin{array}{l}93,02 \\
55,81\end{array}$ & $\begin{array}{l}37,19 \\
30,57\end{array}$ & $\begin{array}{l}0,27 \\
0,17\end{array}$ \\
\hline Moyenne & 68, 37 Faible & 31,64 Faible & 0,19 Peu résistant au choc \\
\hline $\begin{array}{l}\text { Coefficient de variation } \\
(\%)\end{array}$ & 24,81 & 18,65 & 31,56 \\
\hline $\begin{array}{l}\text { Intervalle de confiance } \\
95 \%\end{array}$ & $68,37 \pm 10,78$ & $31,64 \pm 3,74$ & $0,19 \pm 0,05$ \\
\hline Fraké naturel Congo* & 99 Faible & 42,7 Faible & 0,15 Peu résistant \\
\hline Fraké naturel Gabon* & 107,5 Faible & 47 Moyenne & 0,23 Peu résistant \\
\hline $\begin{array}{l}\text { Fraké naturel } \\
\text { Cameroun* }\end{array}$ & 127,5 Moyenne & 52 Moyenne & 0,27 Peu résistant \\
\hline $\begin{array}{l}\text { Fraké naturel Côte } \\
\text { d'lvoire* }\end{array}$ & 116 Moyenne & 49 Moyenne & 0,33 Peu résistant \\
\hline
\end{tabular}

\footnotetext{
*Source : CTFT, 1986
} 


\section{Ahoba et al., J. Appl. Biosci. 2016 Le bois du frake issu de plantation exprime aussi de bonnes qualités technologiques}

Tableau 7 : Quelques qualités physiques et mécanique du bois de Fraké en fonction de la hauteur de l'arbre

\begin{tabular}{|l|c|c|c|c|}
\hline Niveau de prélèvement & $\mathbf{A}[\mathbf{0}-3 \mathrm{~m}$ ] & $\mathbf{B}[3-6 \mathrm{~m}]$ & $\mathbf{C}[6-9 \mathrm{~m}]$ & Autres [9m et plus] \\
\hline Densité $\left(\mathrm{g} / \mathrm{dm}^{3}\right)$ & 456 très leger & 511 léger & 448 très léger & 439 très léger \\
\hline Compression axiale MPa & 36 faible & 41 faible & 31 faible & 30 faible \\
\hline Flexion statique MPa & 68 faible & 78 faible & 77 faible & 79 faible \\
\hline $\begin{array}{l}\text { Flexion dynamique } \mathrm{K} \quad \mathrm{kg} \\
\mathrm{m} / \mathrm{cm}^{2}\end{array}$ & $\begin{array}{c}0,19 \mathrm{peu} \\
\text { résistant au choc }\end{array}$ & $\begin{array}{c}0,46 \text { résistance } \\
\text { moyenne au choc }\end{array}$ & $\begin{array}{c}0,15 \text { peu resistant au } \\
\text { choc }\end{array}$ & $\begin{array}{c}0,24 \text { peu resistant au } \\
\text { choc }\end{array}$ \\
\hline
\end{tabular}

\section{DISCUSSION}

Cubage des billons: Les Frakés de plantation présentent à 32 ans des dimensions intéressantes pour une exploitation en bois d'œurre. Les grumes peuvent atteindre $21 \mathrm{~m}$ de longueur pour $50 \mathrm{~cm}$ de diamètre, similaires à ceux obtenus par Dupuy en 1986. L'arbre étiqueté Fraké 3 a exprimé les plus forts diamètres aussi bien au gros bout, au petit bout et en valeur moyenne. De telles performances peuvent être dues aux différences pédo-climatiques localisées de la parcelle retenue pour l'étude. Le volume moyen de $1,8 \mathrm{~m}^{3}$ obtenu par arbre est pratiquement identique à celui résultant du tarif volume bois fort $\left(0,16-3,10 D+18,24 D^{2}\right)$ établi pour cette essence par Dupuy en 1986. Dans la limite de validité (10 à $45 \mathrm{~cm}$ ) et pour des diamètres d'exploitabilité de 40 $\mathrm{cm}$, ce tarif permet d'atteindre des volumes de 100 à 200 $\mathrm{m}^{3} /$ ha (Dupuy, 1986) pour une plantation bien conduite. La densité préconisée à la plantation est de 700 pieds /ha, ramenée par trois éclaircies successives ( 3 ans, 5 ans et 8 ans) à environ 100 pieds /ha. Pour un diamètre d'exploitabilité de $60 \mathrm{~cm}$, l'âge d'exploitabilité est de 25 à 30 ans (Dupuy, 1986).

Cotation des billons: Les cotations qualitatives montrent que le billon de Fraké est légèrement courbe avec une section circulaire et un cœur centré. Ces cotations positives, favorables à un bon rendement au sciage, constituent un atout intéressant pour la valorisation en bois d'œuvre du Fraké de plantation. Des cotations similaires ont été rapportées par Edi (2000) sur Hopea odorata (Sao), une essence originaire d'Asie, qui présente des conformations et des dimensions comparables à celles du Fraké de plantation.

Sciage des billons: La répartition des débités par largeur fait ressortir pour $56 \%$ d'entre eux, des largeurs supérieures ou égales à $15 \mathrm{~cm}$ contrairement à une essence comme le teck qui ne peut en fournir que $18 \%$ (Sitbon, 1981). Ces largeurs qui correspondent aux dimensions les plus demandées dans les échanges commerciaux, peuvent donner entièrement satisfaction aux exigences du marché général. En y ajoutant les 10\% de complément de largeur accepté par les négociants en bois, ce sont environ $70 \%$ de parts de marché que l'on peut satisfaire par les débités de Fraké. Les produits issus du sciage de Fraké de plantation peuvent, en termes de dimensions, s'insérer sans difficulté sur le marché général.

Rendement sciage: Les rendements obtenus sont similaires à celui de l'Hoppea odorata (Ahoba, 2012) et se rapprochent de ceux de certaines essences de forêt naturelle comme le Guibourtia ehie (Amazakoué) et le Distemonanthus benthamianus (Movingui) sciées en plots. Pour ces essences, des valeurs respectives de $71,4 \%$ et de $76,1 \%$ ont été rapportées par Bigotte (1977).

Aspect du bois : Le bois d'aspect similaire à celui de forêt naturelle est blanc crème avec des figurations décoratives pour les sciages sur dosse. L'aspect "Fraké bariolé" particulièrement apprécié par les consommateurs n'est pas exclusif aux Frakés de forêt naturelle. Ceux de plantation en présentent largement avec diverses formes esthétiques parcourant la longueur de certaines planches.

Qualités physiques et mécaniques: En qualité physique et mécanique, les Frakés de plantation, montrent une tendance à la baisse notamment de la densité du bois en fonction de la hauteur à partir de $6 \mathrm{~m}$. Cette tendance porte à croire que les billes de base donnent du bois de meilleure qualité notamment en densités par rapport aux surbilles. Ces densités peu sensibles aux variations d'humidité, sont inférieures à celles des Frakés de forêt naturelle $\left(550 \mathrm{~g} / \mathrm{dm}^{3}\right)$ et les classent plutôt dans la catégorie des bois très légers. Cependant, au Congo et au Gabon, avec des densités respectives de $450 \mathrm{~g} / \mathrm{dm}^{3}$ et de $480 \mathrm{~g} / \mathrm{dm}^{3}$ rapportées par le CTFT (1986), les Frakés de forêt naturelle se classent dans la même catégorie que les Frakés de plantation. Les retraits totaux sur le volume sont moyens (Durand et Edi, 1979) et le bois sec à l'air varie peu en volume quand l'humidité change ; le bois dans ces conditions est qualifié de " peu nerveux". Ces caractéristiques physiques sont similaires à celles obtenues par Durand et Edi en 1979 sur des Frakés de plantation de 14 et 16 ans. Les gammes de densité observées par ces auteurs 


\section{Ahoba et al., J. Appl. Biosci. 2016 Le bois du frake issu de plantation exprime aussi de bonnes qualités technologiques}

vont de 380 à $420 \mathrm{~g} / \mathrm{dm}^{3}$ contre 368 à $507 \mathrm{~g} / \mathrm{dm}^{3}$ dans notre cas ; les qualifications conformément à la norme AFNOR (1988) demeurent toutefois les mêmes. Les contraintes admissibles en compression axiale ont également tendance à décroitre avec la hauteur de l'arbre. Les valeurs moyennes obtenues font des Frakés de reboisement, des bois faiblement résistants par rapport aux Frakés de forêt naturelle (49 $\mathrm{MPa}$ ) mais cela ne limite en rien leur aptitude à répondre aux usages courants du bois de Fraké. Les résistances en flexion statique sont faibles pour les Frakés de reboisement et

\section{CONCLUSION}

Nous alléguions que les bois de Fraké naturel et artificiel sont comparables par rapport à leurs qualités technologiques. Effectivement, en dépit de quelques qualités physiques et mécaniques minorées dans le bois de Fraké issu de plantation artificielle, les qualifications des 2 types de bois sont pratiquement comparables. En

\section{REFERENCES BIBLIOGRAPHIQUES}

Afnor, 1988. Bois et liège- Recueil de normes françaises $2^{2 e ̀ m e ~ e ́ d i t i o n . ~ E d i t e ́ ~ p a r ~ l ' A s s o c i a t i o n ~ F r a n c ̧ a i s e ~ d e ~}$ Normalisation, $606 \mathrm{p}$.

Ahoba A, Edi K, Coulibaly S. 2012. Sciage - rendement sciage - Classement des débités de Sao (Hopea odorata). Rapport scientifique interne, CNRA, $52 \mathrm{p}$.

Bigotte B. 1977a. Essai de rendement industriel de sciage de l'Amazakoué. Rapport scientifique interne, Centre Technique Forestier TropicalCôte d'Ivoire, $17 \mathrm{p}$.

Bigotte B. 1977b. Essai de rendement industriel de sciage du Movingui. Rapport scientifique interne, Centre Technique Forestier TropicalCôte d'Ivoire, $17 \mathrm{p}$.

CTFT 1986. Liste des propriétés physiques et mécaniques par nom scientifique, par pays et par essai, p 231

Dupuy B. 1986. Principales règles de sylviculture pour les plantations à vocation bois d'œuvre. Centre Technique Forestier Tropical-Côte d'Ivoire, 112 p.

Durand P.Y. 1978. Propriétés physiques et mécaniques des bois de Côte d'Ivoire- Moyennes d'espèce et variabilité intraspécifique. Centre Technique Forestier Tropical-Division Technologie et Préservation Abidjan Côte d'Ivoire, $67 \mathrm{p}$.

Durand P.Y. et Edi K. 1979. Propriétés physiques du Fraké (Terminalia superba)-Plantations de Mopri 1964 et Gonaté 1961 (Résultats des essais moyennes pour les Frakés de forêt naturelle en Côte d'Ivoire (116 MPa) et au Cameroun (127 MPa) à titre indicatif. Par contre au Gabon (107,5 MPa) et au Congo (99 MPa) les résistances en flexion statique rapportées par le CTFT (1986) sont similaires à celles des Frakés de plantation. En résistance sous des charges brusquement variables, les Frakés de reboisement apparaissent comme des bois résilients ou moyennement résilients supportant bien les chocs comme leurs homologues de forêt naturelle.

effet, les Frakés naturels et artificiels de plantation présentent des dimensions et des conformations qui rendent le sciage facile et donnent des rendements intéressants. En conséquence, les Frakés artificiels constituent un atout important dans leur utilisation en bois d'œuvre.

n¹12-01 et 112- 02). Centre Technique Forestier Tropical, Division Technologie et Préservation Abidjan-Côte d'Ivoire, $15 \mathrm{p}$.

Edi K.A. 1999. Note sur Hopea odorata Roxb (Sao). Une essence à promouvoir en plantation. SODEFOR - Direction Technique - S/Direction Recherche et Ecologie, $10 \mathrm{p}$.

Edi K.A. 2000. Essai préliminaire de sciage du bois d'Hopea odorata (Sao). Parcelle Anguédédou 1979. SODEFOR - Direction Technique S/Direction Recherche et Ecologie, $15 \mathrm{p}$.

Sallenave P. 1955. Propriétés physiques et mécaniques des bois tropicaux de l'Union Française, Nogent -sur-Marne, France, Centre Technique Forestier Tropical, $126 \mathrm{p}$.

Sallenave P. 1964. Propriétés physiques et mécaniques des bois tropicaux- 1er supplément, Nogent sur - Marne, France, Centre Technique Forestier Tropical, $79 \mathrm{p}$.

Sallenave P. 1971. Propriétés physiques et mécaniques des bois tropicaux $-2^{\text {ème }}$ supplément, Nogent sur-Marne, France, Centre Technique Forestier Tropical, $123 p$.

Sitbon R. 1981. Etude du sciage de trois(3) lots de teck. Rapport scientifique interne CTFT Côte d'Ivoire, $10 \mathrm{p}$.

Sodefor 2012. Ensemble des reboisements gérés par la Sodefor. Document interne de travail/Direction Technique/Sous-Direction Reboisement, $4 \mathrm{p}$. 